\title{
CAUSTIC WAVES IN GALAXY DISKS \\ PRODUCED IN COLLISIONS WITH LOW MASS COMPANIONS
}

\author{
Curtis Struck-Marcell \\ Astronomy Program, Physics Dept. \\ Iowa State University
}

At this meeting much attention has been focussed on interactions and mergers between roughly equal mass galaxies. On the contrary, I will begin by mentioning a few justifications for studying collisions with relatively low mass companions, specifically, less than about one third of the mass of the target galaxy. The first is simply that such collisions are likely to be common, given that the galaxy luminosity function is broad. The second reason is that such collisions have evidently been less well studied than collisions between nearly equal partners. However, there are a few important exceptions to this generalization, including the sinking satellite problem (e.g. Quinn and Goodman 1986), and the collisional model for the formation of shell galaxies in which a companion of negligible mass is completely disrupted(e.g. Dupraz and Combes 1986, Hernquist and Quinn 1988). The third, and potentially most important reason, is that the effects of a collision with a low-mass companion are less extreme (at least from the big galaxy's point of view!). Thus, these effects are closer to the theorist's ideal of a "small perturbation". This is important for both conceptually understanding the effects of the collision, and for justifying the use of approximate numerical techniques (e.g. restricted three-body) to study them.

The primary effect of such collisions on a target galaxy with a "cold", disk component is the generation of waves in the disk. Here I will focus on the purely stellar waves in such disks. Even

within these limitations a large variety of transient wave morphologies, besides the well-known spirals and rings, are possible. Such nonlinear stellar waves are caustic surfaces, and I believe that singularity or catastrophe theory can be helpful in understanding their properties. (For an excellant introduction see Arnold (1986)). In persuing this understanding we can follow the lead of Arnold, Shandarin, and Zeldovich (1982) who haye applied singularity theorry to the study of two and three dimensional "pancaking" in galaxy formation theory.

The word "caustic" has a variety of specific definitions. For present purposes it suffices to define a caustic as a (compact) zone of stellar orbit crossings, or a zone where two or more collisionless star streams intersect. This idea of orbit crossings immediately harkens back to the kinematic models of Lynds and Toomre (1976) for stellar rings, and Kalnajs' (1973) rotating ellipses model for spiral waves. Because of its cylindrical symmetry, the ring galaxy case provides a particularly simple example that is worth reviewing. 
Figure 1 shows a phase plane cut illustrating ring structure and outward propagation. (The details of the specific kinematic calculation are not important here.) Before the collision we assume that all the stars were in circular orbits, with a negligible thermal velocity component, and zero systematic radial velocity. At times shortly after the central impact all the stars fall inward (negative radial velocity) in response. After the companion moves away from the disk the stars no longer feel the pull of the extra mass towards the center, so centrifugal force reverses the infall, and the stars will move back out, ultimately overshooting their equilibrium radius. For most reasonable disk potentials the epicyclic oscillation period will be shorter in the center, so rings will form there and propagate outward. Some time after ring formation, the innermost stars begin a second cycle of infall. Figure 1 shows a later time when the first ring has propagated well out into the disk and a second ring has formed.

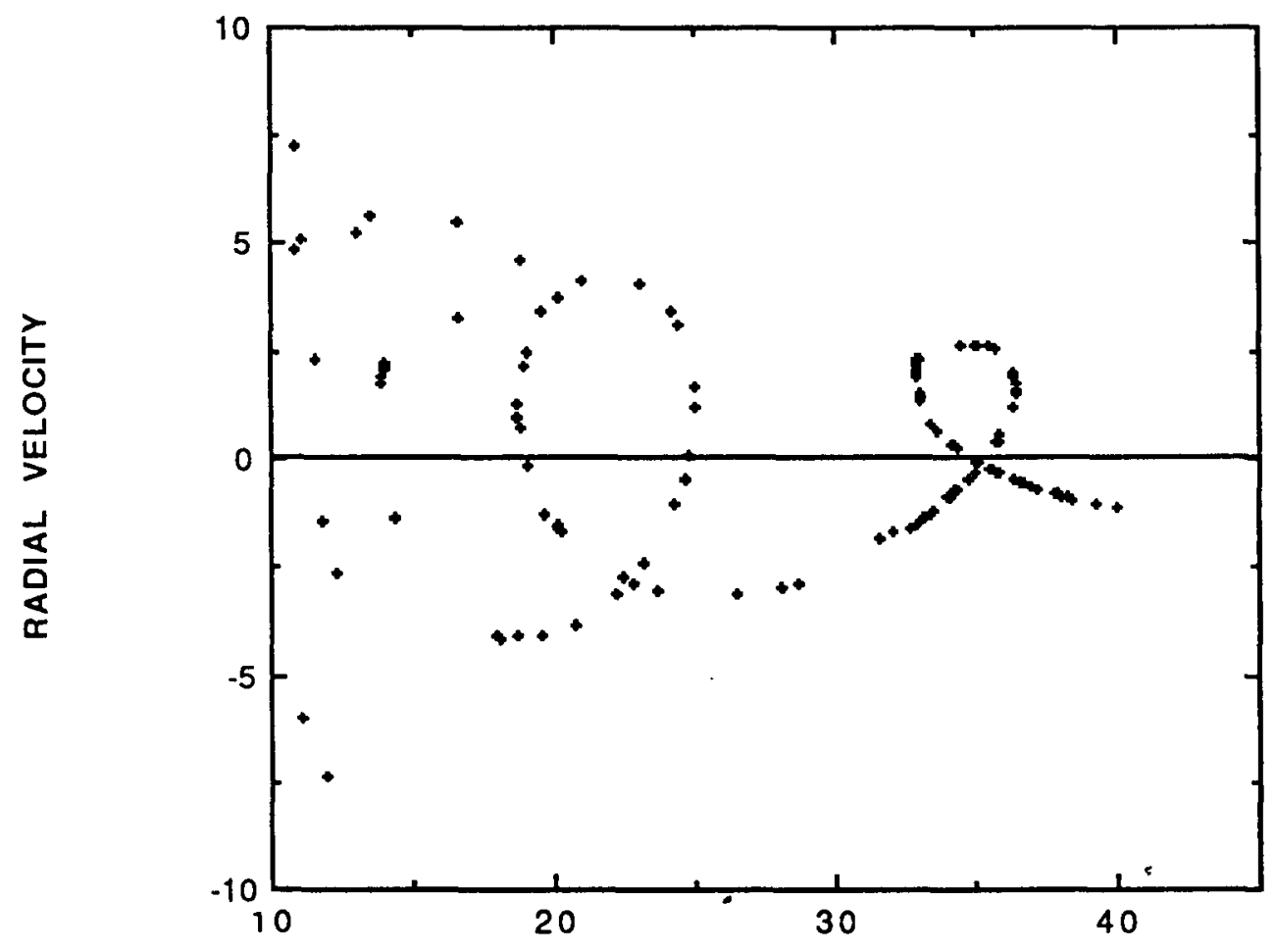

RADIUS

Fig. 1. Phase plane surface of section with dimensionless radial velocity vs. dimensionless radius plotted for particles within a narrow azimuthal range along a radius vector. The results are from a simple kinematical calculation of a ring-making collision. The two loops in the plot correspond to the two stellar rings that have formed by the time shown. 
The rings appear as loops in these $r-v_{r}$ phase space cuts. (N.B. crossings in the phase cut do not represent crossings in the four dimensional phase space, so there is no violation of Louisville's theorem.) A vertical line in the last frame shows that within the loop (ring) there are three interpenetrating star streams, satisfying our definition of caustic. Other verticals show that the ring edges are fold caustics, i.e. projections of a phase space fold onto the disk plane. In fact, such diagrams show that circular stellar rings are born as phase space cusps, and degenerate into paired fold caustics (loops) immediately thereafter. This is a standard sequence of caustic metamorphosis, and is locally the same as Zeldovich pancake formation.

Many ring properties can be calculated with the kinematic model illustrated in Figure 1, including: ring center positions, and the widths and mean densities of rings as a function of time. The idea of these calculations is as follows. If we adopt the idealization that the kinematic equations describe a continuous, collisionless stellar fluid, then these equations predict the occurance of an infinite stellar density at a caustic edge. The density singularity condition can be solved to determine which stars (identified by their initial unperturbed positions) lie within the caustic at any time. The kinematic orbit equation then specifies the location of the caustic edge. At least qualitatively the results seem to agree well with the few high-resolution N-body results available to date, for details see Struck-Marcell and Luban-Lotan (1990).

When we extend our consideration to off-center impacts new, and more complex caustics emerge, including the so-called swallowtail, and Arnold's pocket or purse (hyperbolic umbilic). As before, we can demonstrate the development of these caustics with a simple kinematic model, although in asymmetric cases it is very difficult to derive analytic results (see Struck-Marcell 1990). Figure 2, which is based on a numerical kinematic calculation, shows an example of a swallowtail caustic produced in a slightly off-center collision. At the top is a gray-scale representation of the stellar density in the disk (the range of densities is about an order of magnitude), which shows the high-density central region of the caustic and the several arms radiating out from it. It is still possible to see the familial relationship between this form and rings, or ring plus spirals. This is also true in the phase space cut at the bottom of Figure 2, where loops are again very evident. The swallowtail must appear as something like a bow-tie in the phase cut, since it is characterized by five intersecting star streams. Similarly, family resemblances aside, the swallowtail should be recognizable on the sky. Just on the basis of appearance, NGC 3145, a Hubble Atlas galaxy (Sandage 1961), is a possible candidate. It has not to my knowledge been cited as a possible collision remnant before, but it does have a companion (NGC 3143) with a similar redshift within about 5-6 diameters. Other possible examples of higher order caustics are given in Struck-Marcell (1990). Higher order caustics are also evident in most published high resolution restricted three-body or $\mathrm{N}$-body simulations of interactions. 

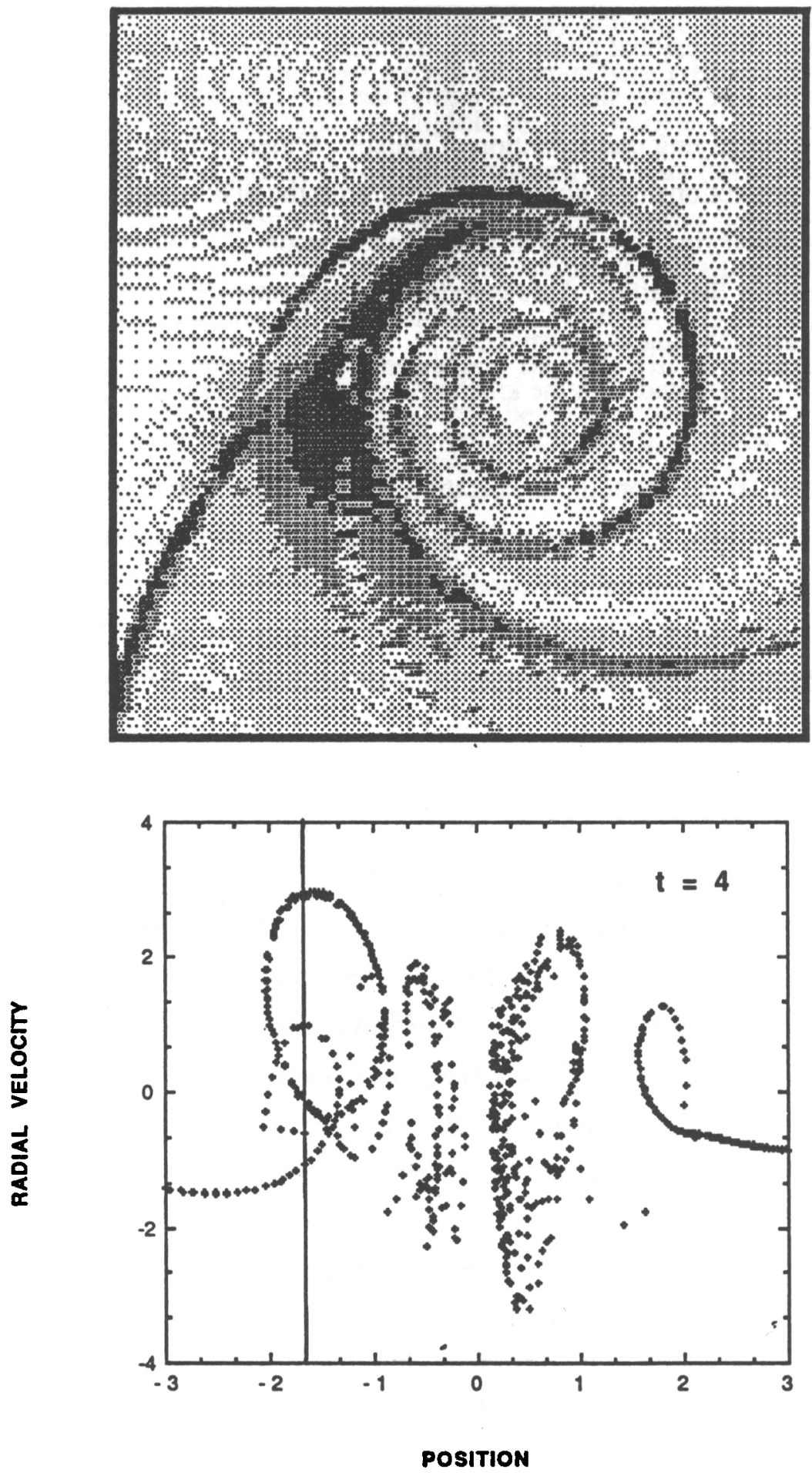

Fig. 2. Top : Gray-scale representation of the surface density of a swallowtail caustic. The gray-scale levels vary over a range of about an order of magnitude in surface density. Bottom: Phase plane section as in Figure 1, but for a slightly off-center collision, and with particles from a strip in both the positive and negative directions along the $x$-axis. The vertical line cuts the five star-streams that make-up a swallowtail caustic. 
The stellar rings and the swallowtail examples suggest several specific ways in which the mathematical theory of caustics and singularities can be helpful in the study of colliding galaxies. These are not unique cases, and so I will conclude with a brief list of tentative answers to the question - why caustics? Possibly the most important reason, but also the least well-defined, is that caustics are a valuable conceptual tool. For example, it is easy to visualize morphological features like a flat surface density profile across a wave in terms of the superposition of several roughly uniform star streams. It is also easy to understand sharp boundaries of over-dense regions as folds in phase space.

A related point is that since there are only a relatively small number of generic caustic forms in two and three dimensions, these forms can provide the basis of a classification scheme for collisional disturbances. The caustic forms are sufficiently distinct that it should be possible to search for them automatically. However, caustics do not always appear in isolation, they can overlap each other, which would complicate such a search. At the same time, the structure of multiple caustics in a galaxy might provide enough information to allow the determination of the interaction parameters fairly uniquely. Moreover, the evolutionary disintegration of caustics by shear and phase mixing may facilitate dating by morphology in collisional systems.

Singularity theory suggests some specific observational checks. High-resolution spectra of a swallowtail morphology should reveal either the multiple star streams, or at least very broad spectral lines relative to surrounding regions.

Finally, at least in symmetric cases, singularity theory provides a method for (semi-)analytic calculation of nonlinear wave structure.

NASA grant NAGW 1009 is gratefully acknowledged for partial support of this work.

\section{REFERENCES}

Arnold, V.I.(1986), Catastrophe Theory, 2nd English edition (New York: Springer-Verlag).

Arnold, V.I., Shandarin, S.F. and Zeldovich, Ya. B.(1982), Geophys. Astrophys. Fluid Dynamics 20, 111.

Dupraz, C., and Combes, F. (1986), Astron. Astrophys. 166, 53.

Hernquist, L., and Quinn, P. J. (1988), Astrophys. J. 331, 682.

Kalnajs, A. J. (1973), Proc. Astron. Soc. Aust. 2, 174.

Lynds, R., and Toomre, A. (1976), Astrophys. J. 209, 382.

Quinn, P. J., and Goodman, J. (1986), Astrophys. J. 309, 472.

Sandage, A. (1961), The Hubble Atlas of Galaxies (Carnegie Inst.: Washington DC).

Struck-Marcell, C. (1990), Astron. J. 99, 71.

Struck-Marcell, C., and Luban-Lotan, P. (1990), Astrophys. J., in press. 